\title{
Post-Adjuvant Therapy
}

National Cancer Institute

\section{Source}

National Cancer Institute. Post-Adjuvant Therapy. NCI Thesaurus. Code C158707.

Therapy administered after to the initial therapy. 